\title{
Three-body collapse for Tabakin potentials and the Thomas effect
}

\author{
A. Delfino \\ Department of Physics and Astronomy, University of Maryland, College Park, Maryland 20742 \\ and Departamento de Física, Universidade Federal Fluminense, 24210 Niterói, Rio de Janeiro, Brasil \\ Sadhan K. Adhikari \\ Instituto de Física Teorica, Universidade Estadual Paulista, 01405 São Paulo, São Paulo, Brasil \\ Lauro Tomio \\ Instituto de Física Teorica, Universidade Estadual Paulista, 01405 São Paulo, São Paulo, Brasil \\ and School of Physical Sciences, The Flinders University of South Australia, Bedford Park, SA 5042, Australia \\ T. Frederico \\ Instituto de Estudos Avançados, Centro Tecnico Aeroespacial, 12225 São José dos Campos, São Paulo, Brasil
}

(Received 16 March 1992)

\begin{abstract}
A system constituted of three bosons interacting via two-body separable potentials with fixed twoboson binding is known to lead to bound-state collapse in the case where the potential parameters allow two-boson $S$-matrix poles close to (resonance) and on (continuum bound state) the real momentum axis. The collapse is shown to be accompanied by an increase in the average kinetic energy of the two-body bound state, which signals a decrease in the range of the two-body interaction for fixed two-body binding. The collapse is claimed to be a manifestation of the well-known Thomas effect which leads to a collapse of the three-body system when the range of the two-body interaction goes to zero for a fixed two-body binding.
\end{abstract}

PACS number(s): 21.45.+v, 03.65.Nk

\section{INTRODUCTION}

Separable interactions have been frequently used in various areas of physics, such as particle, nuclear, and atomic physics, because of the numerical simplicity they bring to the two- and three-body problems [1]. In spite of being successfully employed in three-nucleon $(3 N)$ bound-state and scattering calculations, the usual nucleon-nucleon $(N N)$ separable potentials have the drawback of being unrealistic in yielding $N N$ phase shifts which do not change sign, in contradiction with experiment, at higher energies. With a view to finding a remedy for the above problem Tabakin constructed a rankone separable $N N$ potential with a two-term form factor, capable of reproducing $S$-wave $N N$ phase shifts up to moderately high energies where these phase shifts become negative in agreement with experiment. The use of the Tabakin potential [2] in $3 N$ bound-state calculation, however, leads to a $3 N$ ground state of unusually large binding of few hundred MeV's in addition to a weakly bound excited state. This puzzling result has been studied by several groups of workers [3-5]. Many other similar (nonlocal) potentials were employed with an objective to pinpoint the property of the potential that is responsible for this peculiar behavior. They all associate such a bound-state collapse (BSC) with the appearance of a twobody $S$-matrix pole on-continuum bound state (CBS) [6] - or near (resonance) the real energy axis. It should be recalled that in all such studies the two-body energy is maintained fixed.
An $S$-matrix pole on the real energy axis is a zerowidth resonance and is usually called a CBS. The association with the bound state lies on the fact that the corresponding wave function has no outgoing propagation. It is in fact a localized function of momentum $\phi(p)$ and its Fourier transform $\phi(r)$ decreses exponentially like a bound-state wave function.

This two-body $S$-matrix pole has been conjectured to be responsible for the BSC. The collapse becomes more drastic, contrary to expectations, as the CBS pole moves to higher energies. No satisfactory explanation of this surprising effect has, however, been given. Though this unexplained behavior first appeared in a model nuclear problem it is now generally accepted that there should be a universal mechanism leading to this effect. The objective of the present study is to shed light on the quantum mechanical origin of this phenomenon.

Quite some time ago, while modeling two- and threenucleon problems using very short-range $N N$ potentials with fixed $N N$ binding, Thomas observed that the $3 N$ system collapses as the range of the $N N$ potential tends to zero. As a general quantum mechanical effect the Thomas effect [7] could be stated as follows. If three bosons interact via a short-range pair potential $-\lambda V(r)$ leading to a fixed pair binding and characterized by a range parameter $r_{0}$, then as $r_{0}$ tends to zero, the binding energy of the three-boson ground state increases beyond any limit. However, as $r_{0}$ tends to zero the parameter $\lambda$ has to be increased so that the binding energy of the two-boson system remains fixed. 
In the present study we show that the BSC is a manifestation of the Thomas effect. In order to establish an equivalence between the two phenomena we need to understand the meaning of the range parameter $r_{0}$ introduced in relation to the Thomas effect. For a oneterm local potential such as the exponential, Gaussian, and Yukawa potentials or a one-term separable potential such as the Yamaguchi potential the interpretation of the range is unambiguous as there is only one characterisic range parameter in these potentials. But for a general potential which has two (or more) characteristic range parameters, denoting attraction and repulsion, as in the case of the Tabakin potential discussed above, the definition of $r_{0}$ is not straightforward. We suggest the average kinetic energy $\langle T\rangle$ of the two-body bound state as an indicator of the range of the interaction $r_{0}$ for fixed two-body binding. As the range parameter $r_{0}$ of the Thomas effect tends to zero the average kinetic energy $\langle T\rangle$ tends to infinity. It is easy to convince this with a model $\delta$ function potential, or a separable Yamaguchi potential where $\langle T\rangle$ serves as a more precise measure of the range of the potential when the two-body binding is maintained constant. Consequently, at the onset of the Thomas effect $\langle T\rangle$ diverges and vice versa.

The BSC is always shown to be accompanied by an increase in $\langle T\rangle$, which signals a decrease in the range of the two-body interaction, and suggests that the BSC is a manifestation of the Thomas effect.

Throughout this study the two-body binding, $\gamma^{2}$, is held fixed. As $\langle T\rangle+\langle V\rangle=-\gamma^{2}$, where $\langle V\rangle$ is the average potential energy of the two-body bound state, one could also, equivalently, use $\langle V\rangle$ as a measure of the range of the potential tending towards zero. At the onset of the Thomas effect as the range of the potential tends to zero, $\langle T\rangle \rightarrow \infty$, and $\langle V\rangle \rightarrow-\infty$. However, in this study we shall only be using $\langle T\rangle$ as a measure of the range of the potential.

It is now relevant to comment on some of the interesting features of the BSC. Rupp, Streit, and Tjon [5] (RST) considered a slightly different version of the Tabakin potential in a relativistic approach and made a careful analysis of BSC under two different situations. First, they did not allow the CBS pole to become a resonance in the complex energy plane and then they allowed the CBS pole to become a resonance. In both cases they maintained the two-body binding fixed and studied the evolution of the three-boson binding $B$ with the variation of the parameter $\beta$ related to the range of the potential. In the first case the increase of $\beta$ was governed by a scaling property: $B \rightarrow \beta^{2}$. In the second case $B$ increased with $\beta$ beyond any known scale as the $S$-matrix pole moved into the complex energy plane.

Pantis, Fiedeldey, and Sprung [4] (PFS) had confirmed the above observations of RST earlier. In addition, using a partly nonlocal potential with an artificial strong repulsion at short distances PFS had demonstrated the appearance of a CBS without having a BSC. In the present study and in that of RST this latter situation does not arise.

In brief, associated with a two-body CBS one may or may not have a three-body BSC. Also, in the absence of a two-body CBS and with a two-body resonance pole in the complex energy plane one may have a BSC. What leads to a collapse is not completely understood. Nevertheless, as we see further in this work, a BSC could be explained via a short-distance mechanism of nonlocality, also related to the Thomas effect, without necessarily having a CBS.

In the presence of a purely attractive local or nonlocal two-body potential, the three-boson binding $B$ grows as $\beta^{2}$ where $\beta$ is the inverse range parameter: $\beta=1 / r_{0}$. This scale is extremely accurate in the case of a separable attractive Yamaguchi potential as we demonstrate in the present study. However, when the two-body separable potential has an attractive and a repulsive part governed by two range parameters as in a Tabakin-type potential the above scaling breaks down. In both cases $\langle T\rangle$ serves as an indicator of the range of the potential. The larger the $\langle T\rangle$, the smaller is the range of the potential and the larger is $B$, and one is closer to the Thomas effect.

In Sec. II we present the separable potential model that we employ for the study of the BSC. We provide an analytic expression for $\langle T\rangle$ in this case which we use in our study of the BSC. In Sec. III we present our numerical results and discussions relating $\mathrm{BSC}$ and the Thomas effect. Finally, in Sec. IV we present some concluding remarks.

\section{THE MODEL}

The phenomenon of BSC has been observed in the case of many separable two-body potentials of the Tabakin type. A Tabakin-type separable potential is mainly identified by a momentum space form factor $g(p)$ which may change sign as a function of $p^{2}$. Consequently, the twobody phase shift changes sign at higher energies. The phenomenon of BSC is common to all Tabakin-type potentials. The BSC is not only observed in the case of rank-one potentials, it has also been observed in the case of higher rank potentials [4].

In the present study we consider the following $S$-wave two-body potential in the momentum space:

$$
V(p, q)=\lambda g(p) g(q),
$$

with

$$
g(p)=\sum_{i=1}^{2} \alpha_{i} /\left(p^{2}+\beta_{i}^{2}\right)
$$

We consider this simpler potential with attractive and repulsive parts in this study because of its simplicity, which allows us to understand the origin of BSC. The two-body $t$ matrix for this potential is given by

$$
t\left(p, q, k^{2}\right)=\lambda \tau(k) g(p) g(q),
$$




$$
\begin{aligned}
\tau^{-1}(k) & =1-(2 / \pi) \lambda \int_{0}^{\infty} g^{2}(p) p^{2} d p /\left(k^{2}-p^{2}+i 0\right) \\
& =1-\lambda \sum_{i, j=1}^{2} \alpha_{i} \alpha_{j} /\left[\left(k+i \beta_{i}\right)\left(k+i \beta_{j}\right)\left(\beta_{i}+\beta_{j}\right)\right] .
\end{aligned}
$$

For a purely attractive separable potential (1) with $\alpha_{2}=$ 0 the range can be unambiguously related to the parameter $\beta=\beta_{1}$. This can be realized after taking the Fourier transform of the form factor $g(p)$, which yields an exponential decay characteristic of the range $r_{0}=1 / \beta$. However, in the presence of two terms of opposite signs in the form factor denoting a mixture of attraction and repulsion, the definition of the range is not so straightforward. We consider the average kinetic energy $\langle T\rangle$ of the twobody bound state as a measure of the range $r_{0}$ of the potential.

In the purely attractive case as $\beta$ increases, the threeboson binding energy $B$ as well as the average kinetic energy $\langle T\rangle$ increase, signaling a passage to the Thomas effect. The parameter $\beta$ provides a precise measure of the range of the potential.

We shall see that in the case of a partly attractive and partly repulsive separable potential, as in a Tabakin-type potential, none of the parameters $\beta_{i}$ provides a measure of the range of the potential. However, we shall see that $\langle T\rangle$ still provides a good measure of the range of the potential. Even in this case the divergence of $\langle T\rangle$ clearly signals the appearance of the Thomas effect.

For potential (1) the two-body ground-state wave function in the momentum space is given by

$$
\phi(p)=N g(p) /\left(\gamma^{2}+p^{2}\right),
$$

where $\gamma^{2}$ is the two-body binding in units of $\hbar^{2} / 2 m$, where $m$ is the reduced mass of the two-body system. For the two-nucleon system $\hbar^{2} / 2 m=41.47 \mathrm{MeV} \mathrm{fm}{ }^{2}$ and $\gamma^{2}$ is expressed in $\mathrm{fm}^{-2}$. The normalization constant $N$ is determined by the condition

$$
(2 / \pi) \int_{0}^{\infty} \phi^{2}(p) p^{2} d p=1 .
$$

After some straightforward algebra the average kinetic energy $\langle T\rangle$ is given by

$$
\langle T\rangle=x / y
$$

with

$$
\begin{aligned}
x= & \frac{\alpha_{1}^{2}}{\left(\gamma+\beta_{1}\right)^{3}}+\frac{\alpha_{2}^{2}}{\left(\gamma+\beta_{2}\right)^{3}} \\
& +\frac{2 \alpha_{1} \alpha_{2}}{\left(\beta_{1}+\beta_{2}\right)} \frac{\gamma\left(\beta_{1}+\beta_{2}\right)+2 \beta_{1} \beta_{2}}{\left(\gamma+\beta_{1}\right)^{2}\left(\gamma+\beta_{2}\right)^{2}}
\end{aligned}
$$

and

$$
\begin{aligned}
y= & \frac{\alpha_{1}^{2}}{\gamma \beta_{1}\left(\gamma+\beta_{1}\right)^{3}}+\frac{\alpha_{2}^{2}}{\gamma \beta_{2}\left(\gamma+\beta_{2}\right)^{3}} \\
& +\frac{2 \alpha_{1} \alpha_{2}\left(\beta_{1}+\beta_{2}+2 \gamma\right)}{\gamma\left(\beta_{1}+\beta_{2}\right)\left(\beta_{1}+\gamma\right)^{2}\left(\beta_{2}+\gamma\right)^{2}} .
\end{aligned}
$$

In the present study the binding energy $\gamma^{2}$ of the twobody system is always fixed at the value $(2.225 / 41.47)$ $\mathrm{fm}^{-2}$, when we vary the parameters of the two-body potential. This corresponds to a deuteron binding of 2.225 $\mathrm{MeV}$.

\section{RESULTS AND DISCUSSIONS}

With the separable potential (1) we calculated the twoand three-boson binding energies by solving the usual Lippmann-Schwinger and Faddeev equations in a routine fashion [1], respectively.

Let us first consider the simple case of $\alpha_{1}=0$ in potential (1). In this case there is no possibility of a CBS pole to appear. Still we find it illustrative to present and discuss some results in this case. We take $\alpha_{2}=-1$ and vary $\beta_{2}$ from 3 to $9 \mathrm{fm}^{-1}$. The results are shown in Fig. 1 , where we plot the three-boson energy $B$ versus $\beta_{2}^{2}$. We see that as $\beta_{2}$ increases $B \rightarrow \beta_{2}^{2}$. In this case there is a single range parameter $\beta_{2}$ and we can identify the range of the potential $r_{0}$ to be $1 / \beta_{2}$. Then we have as the range $r_{0} \rightarrow 0, B \rightarrow r_{0}^{-2}$, which is expected from the Thomas effect. In this case the average kinetic energy $\langle T\rangle$ of (7) is given by $\langle T\rangle=\beta_{2} \gamma$. At the onset of the Thomas effect, for a purely attractive separable potential, as $r_{0}$ tends to zero, both $B$ and $\langle T\rangle$ increase beyond bound, provided that two-body binding, $\gamma^{2}$, is held fixed.

Next we consider the full potential (1) with $\alpha_{2}=-1$ and $\beta_{1}=1.4 \mathrm{fm}^{-1}$, which allows for the possibility of a CBS to appear. In our study the constants $\alpha_{1}$ and $\beta_{2}$ were varied arbitrarily, and then $\lambda$ was adjusted to yield a fixed two-body binding of $2.225 \mathrm{MeV}$. It is important for us to look at the variation of $\langle T\rangle, B$, and the two-body $S$ matrix pole position when the potential parameters are varied. In Figs. 2(a) and 2(b) we plot $B$ and $\langle T\rangle$ as functions of $\alpha_{1}$ and $\beta_{2}$. We see in these plots that as $\langle T\rangle$ reaches a maximum, $B$ also does so. In Figs. 3(a) and 3(b) we plot $B$ and $\langle T\rangle$ for fixed values of $\alpha_{1}$ and for a continuous variation of $\beta_{2}$.

From Fig. 1 we find that $\beta_{2}$ is the precise parameter to measure the range of the purely attractive potential. The three-boson BSC associated with the Thomas effect is completely governed by $\beta_{2}$. As $\beta_{2}$ increases the three-

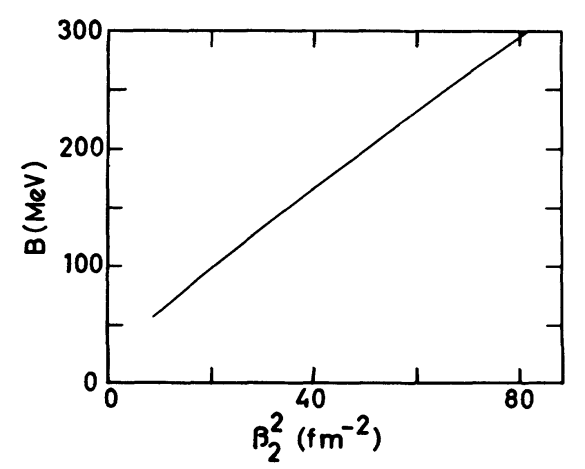

FIG. 1. The three-boson binding $B$ plotted vs $\beta_{2}^{2}$ for a simple Yamaguchi potential. $B$ increases linearly and monotonically with $\beta_{2}^{2}$. 

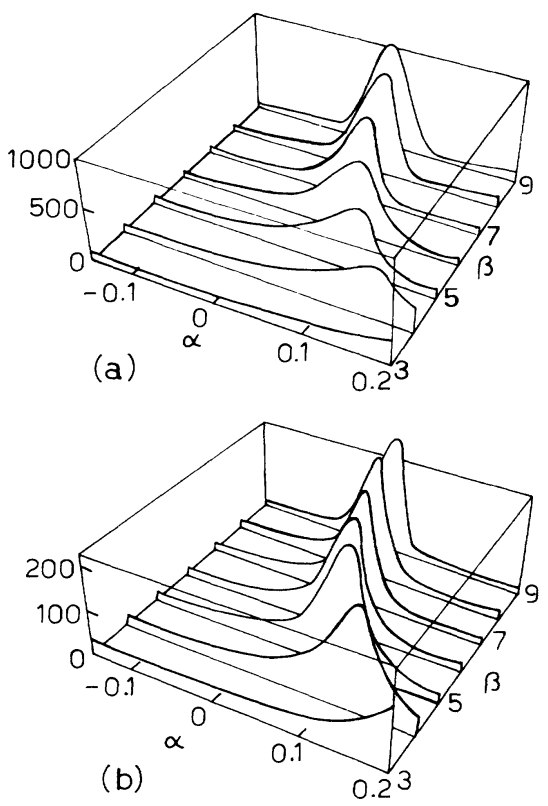

FIG. 2. (a) The three-boson binding $B$ and (b) the average kinetic energy $\langle T\rangle$ of the two-body bound state plotted vs $\alpha_{1}$ and $\beta_{2}$.
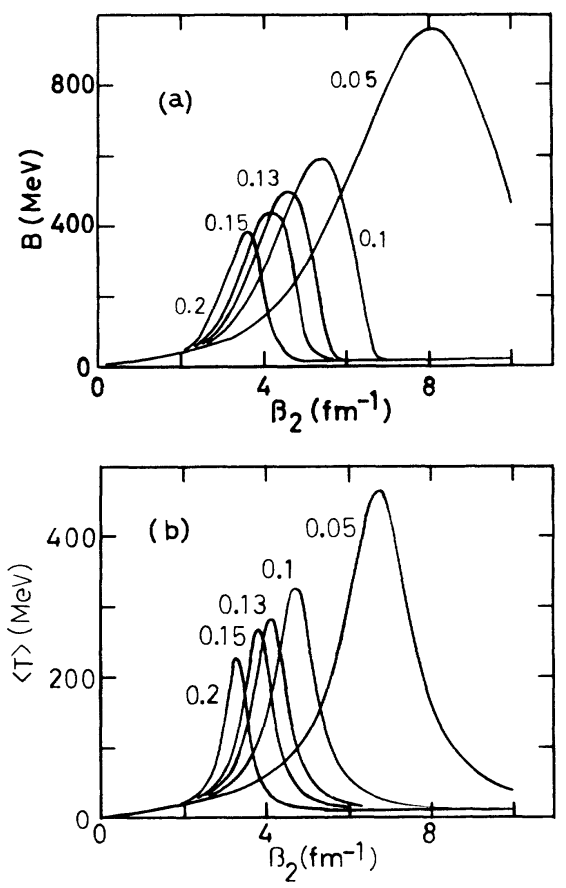

FIG. 3. (a) The three-boson binding $B$ and (b) the average kinetic energy $\langle T\rangle$ of the two-body bound state plotted vs $\beta_{2}$ for fixed values of $\alpha_{1}(=0.2,0.15,0.13,0.1$, and 0.05$)$. The curves are labeled by $\alpha_{1}$. Only one point in each curve corresponds to a CBS. The $\beta_{2}, B$, and $\langle T\rangle$ corresponding to the CBS are given in Table I. The points corresponding to the CBS appear after the maxima of $B$ and $\langle T\rangle$ have been attained in each curve. boson energy steadily increases. This is no longer true in the general case of a partly attractive and a partly repulsive potential when $\alpha_{1}$ is positive and $\alpha_{2}$ is negative. For example, in Fig. 3 for fixed values of $\alpha_{1}$ we find that as $\beta_{2}$ increases both $B$ and $\langle T\rangle$ first increase and then decrease. Naively, one might expect that the increase of $\beta_{2}$ should denote a decrease of the range and a monotonic increase of energy $B$. In this case as there are two characteristic ranges, $\beta_{2}$ alone is not a good indicator of the range. However, we find in this case that $\langle T\rangle$ is a better indicator of the range. From Figs. 2 and 3 we find that first $\langle T\rangle$ increases; and $B$ also increases, then $\langle T\rangle$ decreases and consequently, $B$ also decreases. There is still a shift between the maxima of $B$ and $\langle T\rangle$ which points out that $\langle T\rangle$ is a good, although not perfect, indicator of the range in this case.

Let us now try to understand why $\beta_{2}$ fails to be even a qualitative indicator of the range in the presence of two terms in the form factor of potential (1). Explicitly, the form factor, for $\alpha_{2}=-1$ and $\beta_{1}=1.4 \mathrm{fm}^{-1}$, is written in this case as

$$
g(p)=\alpha_{1} /\left(p^{2}+1.4^{2}\right)-1 /\left(p^{2}+\beta_{2}^{2}\right) .
$$

For a small enough $\alpha_{1}$ the last term in Eq. (10) dominates for a not too large $\beta_{2}$. Then to a first approximation the first term on the right-hand side of Eq. (10) can be neglected and one has a situation similar to the Thomas effect in the case of a purely attractive separable potential. In this case $B$ increases with $\beta_{2}$ which is a good indicator of the range. RST observed this fact in their numerical studies. However, as $\beta_{2}$ increases monotonically for a fixed $\alpha_{1}$, however small, the last term of Eq. (10) becomes negligibly small for a large enough value of $\beta_{2}$. Under this situation the first term on the right-hand side of Eq. (10) dominates and $B$ becomes small and finite characteristic of a range parameter $\beta_{1}=1.4 \mathrm{fm}^{-1}$. This explains qualitatively why $\beta_{2}$ fails to be a proper range parameter for the whole domain of $\alpha_{1}$ and $\beta_{2}$.

Even in the preceding situation $\langle T\rangle$ clearly is a good indicator of the range for two extreme limits of very small and very large $\beta_{2}$. For a very small $\beta_{2}$ the last term in Eq. (10) dominates and as $\beta_{2}$ increases the range of the potential decreases and both $B$ and $\langle T\rangle$ increase. For a large enough $\beta_{2}$ the potential becomes one of a small finite range; consequently, both $B$ and $\langle T\rangle$ become finite. For an intermediate $\beta_{2}$ there is a convenient interpolation between these two extremes. In all cases in Figs. 2 and $3\langle T\rangle$ is a good indicator of the range: whenever $\langle T\rangle$ increases (decreases), $B$ increases (decreases) and vice versa. In Figs. 2 and 3 for very small and large $\beta_{2},\langle T\rangle$ serves as an excellent indicator of range.

At this stage it is interesting to comment on the twobody $S$-matrix pole trajectories in the complex energy (momentum) plane, corresponding to the two extreme values of $\alpha_{1}(=0.2$ and 0.05$)$ of Fig. 3, for fixed $\alpha_{2}(=$ $-1)$ and $\beta_{1}\left(=1.4 \mathrm{fm}^{-1}\right)$, as $\beta_{2}$ is varied. We calculated the two-body $S$-matrix pole trajectory for potential (1) using the method of Ref. [8]. In Fig. 3(a), for a fixed $\alpha_{1}$, as $\beta_{2}$ increases, this $S$-matrix pole trajectory is shown in Fig. 4. It is clear that only one point on each trajec- 


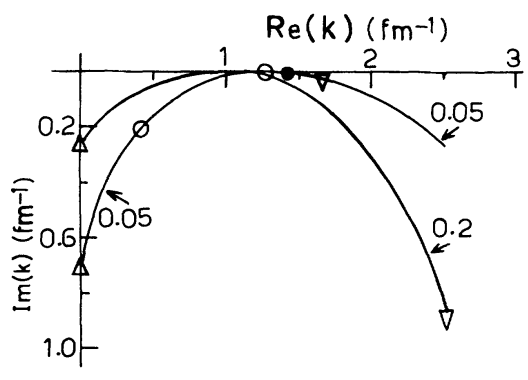

FIG. 4. $S$-matrix pole trajectories labeled by $\alpha_{1}$ in the complex $k$ plane for $\alpha_{1}=0.05$ and $0.2, \alpha_{2}=-1$, and $\beta_{1}=1.4 \mathrm{fm}^{-1}$. Some integral values of $\beta_{2}$ are marked on the trajectories. They are for $\alpha_{1}=0.05, \beta_{2}=6 \mathrm{fm}^{-1}(\triangle), 7$ $\mathrm{fm}^{-1}(\bigcirc), 8 \mathrm{fm}^{-1}(\bullet)$, and $9 \mathrm{fm}^{-1}(\nabla) ;$ for $\alpha_{1}=0.2, \beta_{2}=3$ $\mathrm{fm}^{-1}(\triangle), 4 \mathrm{fm}^{-1}(\bigcirc)$, and $5 \mathrm{fm}^{-1}(\nabla)$.

tory of Fig. 3(a) corresponds to a CBS. All other points of Fig. 3(a) correspond to two-body resonance poles in the complex energy (momentum) plane. So it is obvious that the collapse occurs for a Tabakin-type potential and there is nothing special about the CBS. The CBS is just a point on the Thomas route in direction to BSC. However, unlike in the Thomas effect with a purely attractive potential, the energies $B$ in Figs. 2 and 3 do not go to infinity, as in these cases $\langle T\rangle$ is finite indicating a small, nonzero range $r_{0}$. In this complex situation, with a partly attractive and a partly repulsive potential, though the parameter $\langle T\rangle$ is an indicator of the range, we could not find a simple functional relationship between $\langle T\rangle$ and the range.

In Table I we plot in the pure CBS case the potential parameters $\alpha_{1}, \beta_{2}$, the position of the CBS $p_{c}$, and the corrsponding $B$ and $\langle T\rangle$. The increase of $B$ with $p_{c}$ is confirmed in earlier studies [3-5] as well as in the present one. The increase of $B$ with $\langle T\rangle$ in Table I, and also their subsequent decrease together in Figs. 2 and 3, are consistent with the present interpretation of the BSC as an approximation to the Thomas effect with a small and finite, nevertheless, nonzero $r_{0}$.

Some of the present findings could have been anticipated in the study of RST who calculated an effective local potential equivalent to the Tabakin-type potential and found that in the domain of BSC the equivalent local potential developed a very short-range attractive part. In this short-range local potential picture it would be natural to associate the collapse as a manifestation of the Thomas effect.

TABLE I. The three-boson binding $B$ and average kinetic energy $\langle T\rangle$ of the two-body bound state in the case of CBS with $\alpha_{2}=-1$ and $\beta_{1}=1.4 \mathrm{fm}^{-1}$.

\begin{tabular}{ccccc}
\hline \hline$\alpha_{1}$ & $\beta_{2}\left(\mathrm{fm}^{-1}\right)$ & $p_{c}\left(\mathrm{fm}^{-1}\right)$ & $B(\mathrm{MeV})$ & $\langle T\rangle(\mathrm{MeV})$ \\
\hline 0.05 & 8.47 & 1.307 & 930.5 & 106.7 \\
0.1 & 5.73 & 1.213 & 545.3 & 78.7 \\
0.13 & 4.92 & 1.167 & 446.1 & 70.9 \\
0.15 & 4.53 & 1.149 & 396.3 & 66.3 \\
0.2 & 3.82 & 1.097 & 317.3 & 59.1 \\
\hline \hline
\end{tabular}

\section{CONCLUSIONS}

In this work we have presented for the first time a unified discussion of the Thomas effect and the BSC which occur in three-body systems for some special situations of the two-body interactions. We have suggested that in the situation of collapse the average kinetic energy $\langle T\rangle$ of the two-body bound state is a good indicator of the range of the two-body potential once the two-body binding is maintained fixed. The BSC is demonstrated to be an approximation to the Thomas effect for a two-body potential of short, nevertheless, nonzero range $r_{0}$. In both cases the common feature is maintaining the twobody binding energy fixed. The Thomas effect manifests in a divergent three-boson energy $B$ as the range $r_{0}$ of the potential goes to zero, whereas the BSC occurs for a Tabakin-type separable potential used to simulate the $N N$ interaction.

Other details between the present study and that of RST and PFS remain distinct. This is because three different types of potentials are employed in these studies. Moreover, RST uses relativistic dynamics whereas the other two studies employ nonrelativistic Schrödinger dynamics. For example, the $S$-matrix pole trajectory of these two other studies are distinct from ours. They are qualitatively similar to the one of Fig. 4 but with the direction of the movement of the $S$-matrix pole reversed with the variation of the range parameter. Our pole trajectories, however, become similar to that of RST if we had considered a form factor similar to theirs, e.g., by combining the two terms of Eq. (2), writing the numerator in the form $\left(p^{2}-p_{c}^{2}\right)$, and maintaining $p_{c}$ constant during the movement of the $S$-matrix pole. We have checked this numerically. Also, the potentials of RST and PFS are in some sense much stronger in yielding a very strongly bound three-boson bound state in the domain of collapse. The present model has a weaker potential leading to a less tightly bound three-boson bound state in the situation of collapse.

To summarize, the BSC observed by RST, PFS, and in the present study is an approximation to the Thomas effect for a small nonzero range. The BSC of these studies do not lead to an infinite $B$, because the three bosons in these cases interact via pairwise two-body potentials whose range is quite small but not exactly zero as in a pure Thomas effect. This short-range part of the twobody potential has been established in RST by considering an equivalent local potential. RST, however, did not associate BSC with the Thomas effect. We have established this short-range part of the two-body potential, responsible for the $\mathrm{BSC}$, through a consideration of the average kinetic energy $\langle T\rangle$ of the two-body bound state, which becomes very large in situations of collapse.

\section{ACKNOWLEDGMENTS}

L.T. thanks I. R. Afnan for the kind hospitality at the Flinders University of South Australia and A.D. thanks E. F. Redish for the kind hospitality at the University of Maryland. This work was supported in part by the Conselho Nacional de Desenvolvimento-Científico e Tecnológico ( $\mathrm{CNPq})$ of Brasil. 
[1] S. K. Adhikari and K. L. Kowalski, Dynamical Collision Theory and Its Applications (Academic, Boston, 1991); W. Glöckle, The Quantum Mechanical Few-Body Problem (Springer-Verlag, Berlin, 1983).

[2] F. Tabakin, Phys. Rev. 174, 1208 (1968).

[3] J. E. Beam, Phys. Lett. 30B, 76 (1969); V. A. Alessandrini and C. A. Garcia Canal, Nucl. Phys. A133, 950 (1969).

[4] G. Pantis, H. Fiedeldey, and D. W. L. Sprung, Z. Phys.
A 291, 367 (1979); 294, 101 (1980); referred to as PFS in the text.

[5] G. Rupp, L. Streit, and J. A. Tjon, Phys. Rev. C 31, 2285 (1985); referred to as RST in the text.

[6] B. Bagchi, T. O. Krause, and B. Mulligan, Phys. Rev. C 15, 1623 (1977).

[7] L. H. Thomas, Phys. Rev. 47, 903 (1935).

[8] L. A. L. Roriz and A. Delfino, Phys. Rev. C 38, 607 (1988). 